\title{
Establishment of a Tolerance Budget for the Advanced Photon Source Storage Ring*
}

\author{
H. Bizek, E. Crosbic, E. Lessner, L. Teng \\ Argonne National Laboratory, 9700 So. Cass Avenue, Argonne, IL 60439
}

\section{Abstract}

The limitations on the dynamic aperture of the Advanced Photon Source storage ring due to magnet misalignments and fabrication errors are presented. The reduction of the dynamic aperture is analyzed first for each error considered individually, and then for combined error multipole fields in dipole, quadrupole, and sextupole magnets, excluding and including magnet misalignments. Since misalignments of the strong quadrupoles in the ring induce large orbit distortions, the effects on the dynamic aperture are investigated before and after orbit correction. Effects of off-momentum particles and the tune dependence with momentum are also presented. This extensive analysis leads to the establishment of a tolerance budget. With all the errors set at the tolerance level, and with the orbit distortions corrected, the dynamic aperture reduction is no greater than $50 \%$ of that of the ideal machine.

\section{INTRODUCTION}

The Advanced Photon Source is a 7-GeV synchrotron radiation source designed for high brilliance. Its storage ring has a Chasman-Green lattice, with strong quadrupoles and chromaticity-correcting sextupoles. The sensitivity of the lattice to individual magnet errors was studied extensively, both analytically [1] and by numerical simulation [2], [3]. In this paper we present the results of studies of combined random magnet fabrication and alignment errors and the establishment of error limits based on the extent of reduction of the dynamic aperture.

When alignment and fabrication errors are combined, the dynamic aperture reduction is expected to be large. Due to the strong focusing required by the low emittance, orbit distortions caused by quadrupole displacements can be considerable--the APS storage ring has a large orbit-to-quadrupole displacement ratio $^{1}$, of the order of 50 [4]. Since orbit distortions are mainly caused by dipole strength errors, dipole roil, and quadrupole misalignments, we have analyzed first, the effects of combined errors that do not affect the closed orbit to fu'st order, and then, of all errors together. For the latter group, we determined the dynamic aperture reduction both before and after orbit correction.

\section{MULTIPOLE FIELD ERRORS}

We define multipole ficld errors as those which do not cause closed orbit distortions. Analysis of the lattice responses to variations of a single error strength is straight-forward; not

* Work supported by U.S. Department of Er.ergy, Office of Basic Energy Sciences under Contract No. W-31-109-ENG-38.

${ }^{1}$ Defined as the ratio of the rms quadrupole displacements and the rms orbit distortions. so, when several errors are present. In order to understand the dynamic aperture behavior in the latter case, we studied several levels of error values, starting with every error at its individual tolerance limit $^{2}$ and the subsequent levels with strengths reduced proportionately. The resulting tolerance budget, shown in Table I, represents those values for which, when construction and alignment errors are combined, the extent of dynamic aperture reduction, after correction of closed orbit distortions, is no greater than $50 \%$. In general, combined multipole error tolerance budget limits are smaller than individual tolerance limits by a factor of five to ten. We note that in Table I the multipole cuefficients refer to values at a radius of $1.0 \mathrm{~cm}$, and are normalized such that, for a $2 n$-pole magnet, $b_{n-1}=1.0 \mathrm{~cm}^{-n+1}$, with the magnetic field parametrized by:

$$
B=B_{0} \sum_{n=0}\left(b_{n}+i a_{n}\right)(x+i y)^{n}
$$

We also studied the combined normal and the combined skew multipole errors separately. The combined normal multipole errors alone, set at the budget tolerance limits, produce an average dynamic aperture reduction of about $43 \%$. The corresponding combined skew multipole errors produce an average reduction of about 35\%. Both normal and skew errors together yield a reduction of some $50 \%$, as mentioned. The dynamic aperture in the presence of multipole errors is shown in Figure 1, where the ideal dynamic aperture is also shown for comparison.

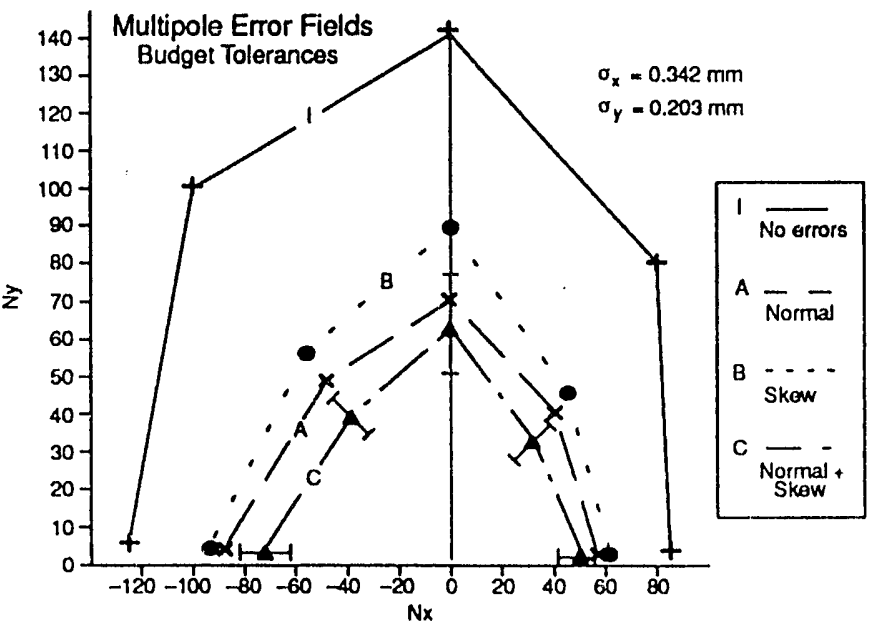

Figure 1

We also studied the combined normal and the combin

Comparison of the dynamic aperture in presence of random normal multipole errors fields (A); random skew multipole crror fields (B); and random normal and skew errors combined (C). Error field components are at the budget tolerances. Axes are in units of the rms beam sizes at the center of the straight section ( $\sigma_{\chi}$ and $\sigma_{y}$ ). The bars represent the error spread over ten machines

${ }^{2}$ Defined as the error strength which reduces the ideal dynamic aperture by $50 \%$. under contract No. W.31-109-ENG.38. Accordingly, the U.S. Government retains a nonexclusive, royalty-tree license to bublish or reproduce the published form of this contribution, or allow others to do so, for U.S. Government nurnoses. 
Table 1 Error Tolerance Budget

\begin{tabular}{|c|c|c|c|}
\hline Error Type & Individual Tol. & Budget Tol. & \\
\hline Roll angle misalignments of dipole magnets & 1.0 & 0.5 & $\mathrm{mrad}$ \\
\hline Horizontal or vertical displacements of quadrupole magnets & 0.1 & 0.2 & $\mathrm{~mm}$ \\
\hline Error dipole fields in dipole magnets & $2 \times 10^{-3}$ & $1 \times 10^{-3}$ & \\
\hline Horizontal or vertical displacements of sextupole magnets & 0.2 & 0.2 & $\mathrm{~mm}$ \\
\hline Normal quadrupole field errors in dipole magnets & $4 \times 10^{-4}$ & $1 \times 10^{-4}$ & $\mathrm{~cm}^{-1}$ \\
\hline Normal sextupole field errors in dipole magnets & $3 \times 10^{-4}$ & $1 \times 10^{-4}$ & $\mathrm{~cm}^{-2}$ \\
\hline Normal octupole field errors in dipole magnets & $3 \times 10^{-4}$ & $1 \times 10^{-4}$ & $\mathrm{~cm}^{-3}$ \\
\hline Error field gradients in quadrupole magnets & $4 \times 10^{-3}$ & $1 \times 10^{-3}$ & $\mathrm{~cm}^{-1}$ \\
\hline Normal sextupole field errors in quadrupole magnets & $1 \times 10^{-3}$ & $2 \times 10^{-4}$ & $\mathrm{~cm}^{-2}$ \\
\hline Normal octupole field errors in quadrupole magnets & $3 \times 10^{-4}$ & $2 \times 10^{-4}$ & $\mathrm{~cm}^{-3}$ \\
\hline Error sextupole field gradients in sextupole magnets & $2 \times 10^{-2}$ & $4 \times 10^{-3}$ & $\mathrm{~cm}^{-2}$ \\
\hline Normal octupole field errors in sextupole magnets & $1 \times 10^{-2}$ & $5 \times 10^{-3}$ & $\mathrm{~cm}^{-3}$ \\
\hline Skew quadrupole field errors in dipole magnets & $7 \times 10^{-4}$ & $1 \times 10^{-4}$ & $\mathrm{~cm}^{-1}$ \\
\hline Skew sextupole field errors in dipole magnets & $2 \times 10^{-4}$ & $3 \times 10^{-5}$ & $\mathrm{~cm}^{-2}$ \\
\hline Skew octupole field errors in dipole magnets & $1 \times 10^{-4}$ & $5 \times 10^{-5}$ & $\mathrm{~cm}^{-3}$ \\
\hline Skew quadrupole field errors in quadrupole magnets & $3 \times 10^{-3}$ & $5 \times 10^{-4}$ & $\mathrm{~cm}^{-1}$ \\
\hline Skew sextupole field errors in quadrupole magnets & $7 \times 10^{-4}$ & $1 \times 10^{-4}$ & $\mathrm{~cm}^{-2}$ \\
\hline Skew octupole field errors in quadrupole magnets & $2 \times 10^{-4}$ & $1 \times 10^{-4}$ & $\mathrm{~cm}^{-3}$ \\
\hline Skew sextupole field errors in sextupole magnets & $2 \times 10^{-2}$ & $2 \times 10^{-3}$ & $\mathrm{~cm}^{-2}$ \\
\hline Skew octupole field errors in sextupole magnets & $2 \times 10^{-2}$ & $2 \times 10^{-3}$ & $\mathrm{~cm}^{-3}$ \\
\hline
\end{tabular}

When momentum errors of $2 \%$ are added to multipole errors, the average reduction is $57 \%$, $5 \%$ more than for on-momentum particles. The horizontal tune shifts by $4 \times 10^{-2}$ and the vertical tune by $-5 \times 10^{-3}$ with respect to the on-momentum particles. The horizontal and vertical tune shifts due to the combined multipole error fields alone are $-1 \times 10^{-3}$ and $-7 \times 10^{-4}$, respectively. The tune variation due to the combined field and momentum errors can be seen in Figure 2.

\section{CONSTRUCTION AND ALIGNMENT ERRORS}

As expected, the dynamic aperture reduces drastically when orbit distortions are added to construction errors. Multipole errors at the budget tolerances combined with orbit distortions resulting from estimated precision obtainable even with the best survey and alignment techniques produced zero dynamic aperture for eight of the ten secds considered. Since closed orbit errors are the main cause of optics distortions, an effective orbit correction mechanism is necessary.

Our simulations of combined errors with orbit distortion correction were performed with the program "RACETRACK with Orbit Corrections" [5] using the 3-magnet bump method. Nine beam position monitors and eight horizontal and six vertical correction dipole magnets are provided in each cell, with the monitors positioned as close as possible to the sextupole mag-

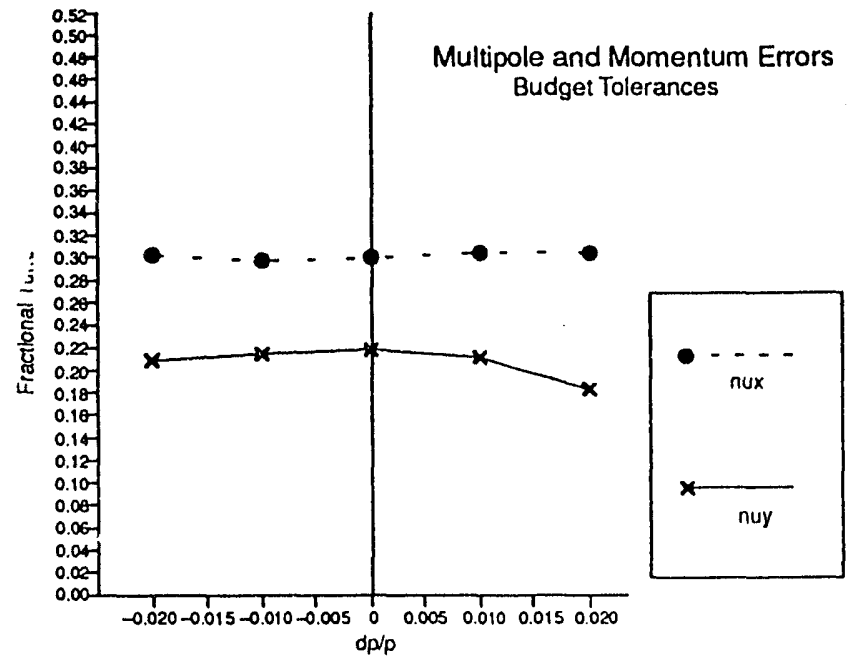

Figure 2

Tune variation with momentum errors in the presence of multipole error fields at the budget tolerances. Only the fractional part of the tunes are depicted.

nets. We employed a total of seven beam position monitors, four horizontal and four vertical corrector magnets per cell.

Random quadrupole displacements of $0.2 \mathrm{~mm} \mathrm{rms,} \mathrm{dipole}$ field strength errors of $1 \times 10^{-3} \mathrm{rms}$ and dipole roll angle misalignments of $5 \times 10^{-4}$ were added to normal and skew random multipoles up to octupole error fields. We also set the resolu- 
tion of the beam position monitors (BPM) $100.2 \mathrm{~mm}$ rms. However, at this level the BPM inaccuracies do not affect the dynamic aperture: if we define the average recovery ratio of the dynamic aperture as the ratio of the dynamic aperture obtained with $0.2 \mathrm{~mm}$ ims beam monitor accuracy to that obtained with perfect monitor accuracy, the recovery ratio for the storage ring is as high as $96 \%$.

After correction, $50 \%$ of the ideal dynamic aperture is recovered, as can be seen in Figure 3. For comparison, we included the reduction for the corresponding errors at the individual tolerance limits. The maximum residual closed orbit distortion is on the order of $0.15 \mathrm{~mm}$ in both planes. The maximum corrector strength distribution has a mean of $0.4 \mathrm{mrad}$, well within the corrector magnet specifications. The corrected orbit functions are also within acceptable values. Figure 4 shows the horizontal closed orbit distortions for a typical seed, before and after correction.

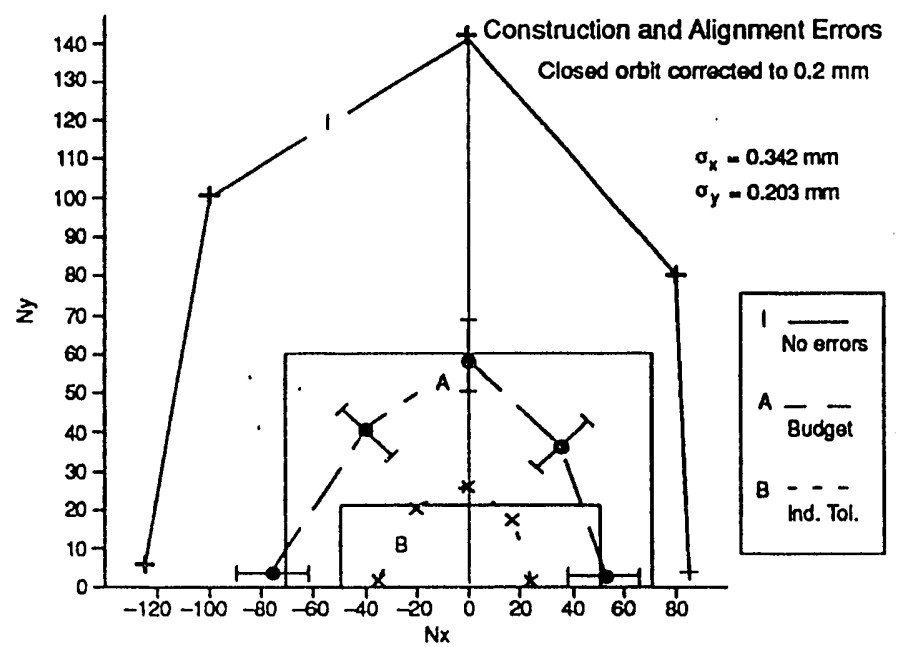

Figure 3

Dynamic aperture in the presence of magnet construction and alignment errors, after orbit corrections to $0.2 \mathrm{~mm} \mathrm{rms}$, for errors at the budget tolerances and at the individual tolerance limits. The physical aperture at the insertion device and elsewhere in the ring are also shown.

\section{CONCLUSION}

With the criterion that a minimum of half of the ideal dynamic aperture be available after orbit corrections, an error tolerance budget for the APS storage ring is established. These tolerances are conservative for all magnets: the most recent set of multipole coefficients measured on the prototype dipole,

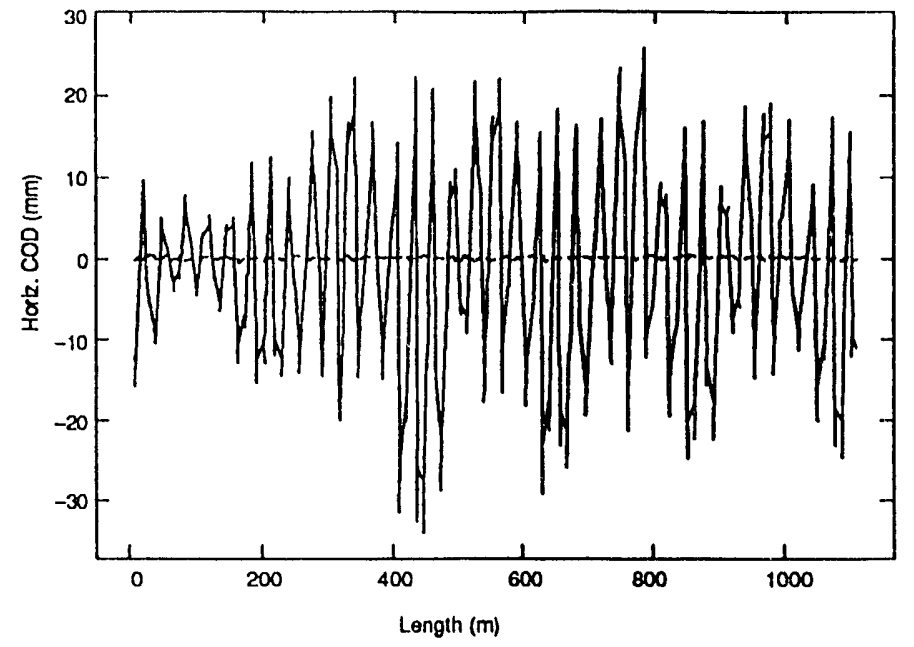

Figure 4

Horizontal closed orbit distortions for a typical seed before and after corrections to $0.2 \mathrm{~mm}$ rms. Magnet field and alignment errors at budget tolerances.

quadrupole, and sextupole magnets are lower than those adopted for the budget by at least a factor of two. Within the budget requirements and with orbit corrections obtained with the corrector system as designed, the dynamic aperture at the insertion devices is still larger than the physical aperture.

The most severe reduction effects on the dynamic aperture of the storage ring come from quadrupole displacements, as expected. However, in the worst case scenario, when all errors are included at the specified tolerance levels and when closed orbit distortions are corrected, the orbit functions and the dynamic aperture are entirely acceptable.

\section{REFERENCES}

[1] L. C. Teng, Argonne National Laboratory, unpublished report (1992).

[2] H. Bizek, E. Crosbie, E. Lessner, L. C. Teng, J. Wirsbinski, "Effects of Errors on the Dynamic Aperture of the Advanced Photon Source Storage Ring," 1991 Particle Accelerator Conference Record, 210-212 (1991).

[3] H. Bizek, E. Crosbie, E. Lessner, L. C. Teng, J. Wirsbinski, "Effects of Construction and Alignment Errors on the Orbit Functions of the APS Storage Ring," 1991 Particle Accelerator Conference Record, 2778-2780 (1991).

[4] E. Crosbie, Argonne National Laboratory, unpublished report (1991).

[5] H. Nishimura, A. Wrulich, "RACETRACK with OrbitCorrections" (1987).

\section{DISCLAIMER}

This report was prepared as an account of work sponsored by an agency of the United States Government. Neither the United States Government nor any agency thereof, nor any of their employees, makes any warranty, express or implied, or assumes any legal liability or responsibility for the accuracy, completeness, or usefulness of any information, apparatus, product, or process disclosed, or represents that its use would not infringe privately owned rights. Reference herein to any specific commercial product, process, or service by trade name, trademark, manufacturer, or otherwise does not necessarily constitute or imply its endorsement, recommendation, or favoring by the United States Government or any agency thereof. The views and opinions of authors expressed herein do not necessarily state or reflect those of the United States Government or any agency thereof. 

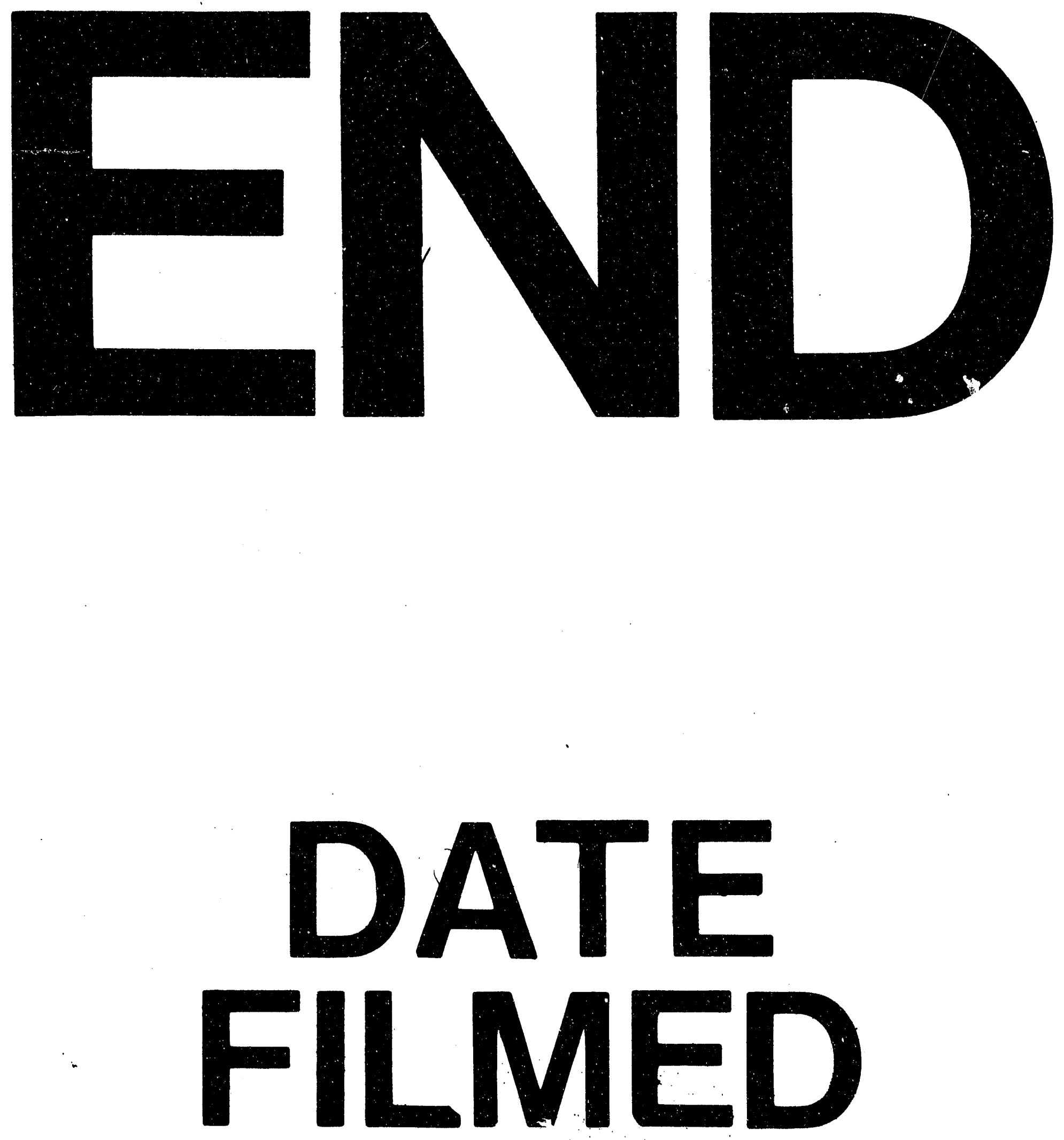

1

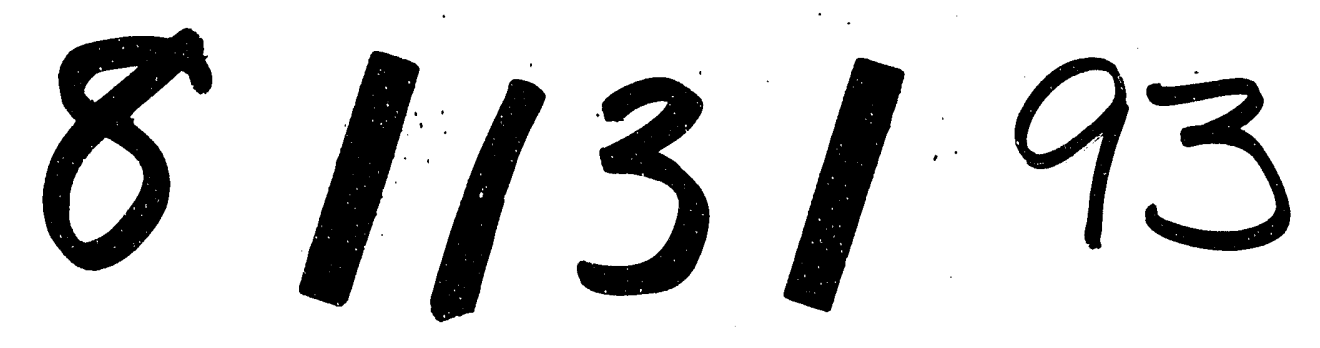


